GUIDELINE UPDATE

\title{
What makes for an effective stop-smoking service?
}

\author{
Leonie S Brose, ${ }^{1}$ Robert West, ${ }^{2}$ Máirtín S McDermott, ${ }^{1}$ Jennifer A Fidler, ${ }^{2}$ \\ Emma Croghan, ${ }^{3}$ Andy McEwen ${ }^{2}$
}

\begin{abstract}
- Additional tables are published online only. To view these files please visit the journal online (http://thorax.bmj. com)

${ }^{1}$ NHS Centre for Smoking Cessation and Training (NCSCT), University College London, London, UK

${ }^{2}$ Cancer Research UK Health Behaviour Research Centre, University College London, London, UK

${ }^{3}$ Department of Health, London, UK
\end{abstract}

\section{Correspondence to}

Dr Leonie S Brose, NHS Centre for Smoking Cessation and Training (NCSCT), University College London, 1-19 Torrington Place, London WC1E 7HB, UK

leonie.brose@ncsct.co.uk

Received 8 February 2011 Accepted 31 May 2011 Published Online First 27 June 2011

\begin{abstract}
Background The English network of stop-smoking services (SSSs) is among the best-value life-preserving clinical intervention in the UK NHS and is internationally renowned. However, success varies considerably across services, making it important to examine the factors that influence their effectiveness.
\end{abstract}

Methods Data from 126890 treatment episodes in 24 SSSs in 2009-10 were used to assess the association between intervention characteristics and success rates, adjusting for key smoker characteristics. Treatment characteristics examined were setting (eg, primary care, specialist clinics, pharmacy), type of support (eg, group, one-to-one) and medication (eg, varenicline, single nicotine replacement therapy (NRT), combination of two or more forms of NRT). The main outcome measure was abstinence from smoking 4 weeks after the target quit date, verified by carbon monoxide concentration in expired air.

Results There was substantial variation in success rates across intervention characteristics after adjusting for smoker characteristics. Single NRT was associated with higher success rates than no medication IOR 1.75, 95\% Cl 1.39 to 2.22); combination NRT and varenicline were more successful than single NRT (OR 1.42, 95\% Cl 1.06 to 1.91 and OR $1.78,95 \% \mathrm{Cl} 1.57$ to 2.02 , respectively); group support was linked to higher success rates than one-to-one support (OR 1.43, 95\% Cl 1.16 to 1.76); primary care settings were less successful than specialist clinics (OR 0.80, 95\% Cl 0.66 to 0.99).

Conclusions Routine clinic data support findings from randomised controlled trials that smokers receiving stop-smoking support from specialist clinics, treatment in groups and varenicline or combination NRT are more likely to succeed than those receiving treatment in primary care, one-to-one and single NRT. All smokers should have access to, and be encouraged to use, the most effective intervention options.

\section{INTRODUCTION}

Tobacco smoking is estimated to lead to 5 million premature deaths each year and is the major preventable cause of lung cancer and chronic obstructive pulmonary disease. Success rates of unaided quit attempts are extremely low at less than $5 \%$. Fortunately, effective forms of help are available including nicotine replacement therapy (NRT), bupropion and varenicline, as well as behavioural support. ${ }^{1}$ In 1999 England became the first country to establish a comprehensive national network of stop-smoking services (SSSs), providing a combination of medication and behavioural support. ${ }^{2}$ These are now among the best-value lifepreserving interventions in the UK NHS. ${ }^{2}$

There are approximately 150 SSSs in England and there is considerable latitude in the kind of intervention offered. A wide variation in success rates has been observed across services. In 2009-10, 4-week biochemically-verified quit rates (the Department of Health standard benchmark for assessing success rates) ranged from 3\% to $58 \%$ (average $34 \%$ ). ${ }^{3}$ Some of the variation will be due to differences in data gathering, recording practices, levels of biochemical verification and client characteristics. However, differences may also be due to variation in the content, type and setting of the intervention provided. Many SSSs record key information about client characteristics and intervention provision that can help us to understand where the sources of variation in performance are arising. Potentially relevant client characteristics include age, gender, level of economic deprivation and degree of nicotine dependence. ${ }^{4}$ The strength of motivation to stop is not frequently measured, but it has been found to have little or no association with success rates in western smokers. ${ }^{5}$ Potentially important intervention factors are: (1) type of medication used, where evidence from randomised clinical trials (RCTs) would lead one to expect that varenicline and combination NRT (patch plus a faster acting form) would be more effective than single form NRT; (2) group versus individual behavioural support, where comparison of efficacy rates across RCTs would suggest that group support would be more effective; and (3) specialist clinic versus pharmacy and primary care settings, where evidence suggests that specialist clinics may be more effective. ${ }^{6}$

Evaluating how intervention characteristics affect effectiveness after adjusting for client characteristics should help to establish optimal service configurations. The findings should help inform intervention provision, both in the UK and internationally, and indicate whether RCT evidence translates into routine practice. The aim of the present study was to assess associations between key aspects of service provision and treatment outcome while adjusting for key smoker characteristics in a large sample of smokers attending English SSSs.

\section{METHODS \\ Sample}

QuitManager (North51, Nottingham, UK) is an online database system for recording information on smoker and intervention characteristics in accordance with the Department of Health's 
standard monitoring requirements. ${ }^{6}$ Twenty-four of the first 40 SSSs to use it agreed to share anonymised data for the current audit. All 'completed treatment episodes' between 1 April 2009 and 30 June 2010 were included as individual records. A completed treatment episode involves a smoker setting a definite quit date with the service and at least 4 weeks having elapsed from that point to the point of data gathering.

\section{Outcome measure}

The outcome measure was successful quitting as defined by the Department of Health - that is, quitters who report 4 weeks after the designated quit date that they have not smoked for at least 2 weeks and a concentration of carbon monoxide (CO) in expired air of $<10 \mathrm{ppm}^{6}{ }^{6}$ As is standard practice, clients lost to follow-up were regarded as smoking. ${ }^{6}$ Long-term quit rates can be estimated reliably from $\mathrm{CO}$-validated 4 -week quit rates and comparative quit rates are stable over short- and long-term follow-ups, ${ }^{6}$ therefore there is a reasonable level of confidence that any associations observed would translate into long-term differences.

\section{Predictor variables: intervention characteristics}

These included medication (no medication, single NRT, combination NRT, bupropion, varenicline), intervention type (one-to-one, drop-in, open group, closed group, telephone support and 'other' such as couple/family sessions) and intervention setting (specialist clinics, primary care, pharmacy and 'other' such as prisons).

\section{Possible confounding factors: client characteristics}

These included age, gender, ethnicity, occupation, exemption from prescription charges (as a proxy measure for economic deprivation) and whether this was a first or subsequent treatment episode. A sensitivity analysis included first treatment episodes only. The level of nicotine dependence (Fagerstrom test for nicotine dependence (FTND): possible range 0-10, higher scores indicating a higher level of dependence ${ }^{7}$ ) was recorded for a minority of clients (6\%), as were cigarettes/day (16\%) and time to first cigarette $(11 \%)$, and so not included in the main analyses. However, dependence was compared across intervention options to determine whether this could explain differences in outcome.

\section{Analysis of data}

SPSS 17.0 and Stata 11.0 were used. Complex samples multiple logistic regression analysis with SSSs defined as clusters was conducted to analyse the extent of differences in success rates between intervention options while taking client characteristics and interdependence within SSSs into account. To assess differences in success rates between SSSs adjusting for other intervention and client characteristics, a separate logistic regression was undertaken with SSS as a predictor variable rather than as a cluster. Mean nicotine dependence was compared across interventions by one-way ANOVA with posthoc pairwise comparisons.

\section{RESULTS}

Analyses were based on 126890 consecutive treatment episodes. Participant demographics and intervention characteristics were similar to those of all clients attending SSSs in England (see table S1 in online supplement). For most clients it was the first treatment episode, about half used combination NRT or varenicline, one-third were seen in specialist clinics and the vast majority received one-to-one support (see table S1 in online supplement). The average $\mathrm{CO}$-verified quit rate was $36.0 \%$ (range 3.8-56.4\%), which was marginally higher than the rate of $33.9 \%$ across all English SSSs. ${ }^{3}$

Those using single NRT had higher success rates than those not using medication. Combination NRT and varenicline had significantly higher odds of success than single NRT (table 1). Group sessions were more successful than one-to-one support; drop-in clinics were the least successful type of intervention. Specialist clinics were more successful than primary care Success rates did not differ between first and subsequent treatment episodes.

In a multiple regression using SSS as an additional predictor rather than a clustering variable, SSS emerged as a highly significant predictor of success after adjusting for client and intervention variables, as in the main analysis $(p<0.001)$. Using the SSS with a success rate closest to the average as the reference (35.5\%), ORs for success ranged from 0.07 (95\% CI 0.06 to 0.08 ) to 2.19 (95\% CI 1.93 to 2.49 ) across the 24 SSSs

Nicotine dependence varied across medication used ( $F$ $(4,7761)=37.91, p<0.001)$; those receiving varenicline (mean (SD) 5.68 (2.15)) or combination NRT (5.52 (2.26)) had significantly $(p<0.001)$ higher dependence than those without medication (4.57 (2.72)) or single NRT (4.88 (2.44)). Clients in specialist clinics $(5.48(2.27))$ were more dependent than those in unspecified 'other' settings (5.12 (2.36), $\mathrm{p}=0.03$ ); other differences across settings were not significant. Dependence differed across intervention types $(\mathrm{F}(5,7660)=11.15, \mathrm{p}<0.001)$; clients receiving one-to-one support had lower scores (5.31 (2.39)) than those in drop-in clinics (5.56 (2.28), $\mathrm{p}=0.001)$ or open groups (5.80 (2.08), $\mathrm{p}<0.001)$.

Table 1 Complex samples (SSS as cluster) multiple logistic regression predicting abstinence $(n=126671)$

\begin{tabular}{llr}
\hline & \multicolumn{2}{c}{ CO-validated 4-week abstinence* } \\
\cline { 2 - 3 } & OR (95\% Cl) & p Value \\
\hline Medication & $1.75(1.39$ to 2.22$)$ & $<0.001$ \\
Single NRT vs no medication & $1.42(1.06$ to 1.91$)$ & 0.019 \\
Combination NRT vs single NRT & $1.12(0.96$ to 1.30$)$ & 0.160 \\
Bupropion (Zyban) vs single NRT & $1.78(1.57$ to 2.02$)$ & $<0.001$ \\
$\quad$ Varenicline (Champix) vs single NRT & & \\
Intervention type (reference: one-to-one) & $1.43(1.16$ to 1.76$)$ & 0.001 \\
$\quad$ Closed group & $0.72(0.57$ to 0.90$)$ & 0.003 \\
Drop-in & $1.46(1.19$ to 1.78$)$ & $<0.001$ \\
Open (rolling) group & - & - \\
Telephone support $\dagger$ & $0.97(0.68$ to 1.38$)$ & 0.851 \\
Other & & \\
Intervention setting (reference: specialist clinics) & $0.80(0.66$ to 0.99$)$ & 0.037 \\
$\quad$ Primary care & $0.94(0.83$ to 1.06$)$ & 0.303 \\
Pharmacy & $0.87(0.69$ to 1.10$)$ & 0.239 \\
Other & $0.99(0.90$ to 1.08$)$ & 0.749 \\
Treatment episode (later vs first) &
\end{tabular}

${ }^{*}$ Adjusted for gender (reference: male; $0 \mathrm{R} 0.93,95 \% \mathrm{Cl} 0.90$ to $0.96, \mathrm{p}<0.001$ ), age (pe year increase: $\mathrm{OR} 1.02,95 \% \mathrm{Cl} 1.01$ to $1.02, \mathrm{p}<0.001$ ), ethnicity (reference: white; NS), occupation (reference: routine and manual; sick/disabled and unable to work: OR 0.62,95\% $\mathrm{Cl} 0.57$ to $0.67, \mathrm{p}<0.001$; never worked/long-term unemployed: $\mathrm{OR} 0.68,95 \% \mathrm{Cl} 0.63$ to $0.74, p<0.001$; full-time student: OR $0.72,95 \% \mathrm{Cl} 0.64$ to $0.81, \mathrm{p}<0.001$; home carer: NS; retired: NS; intermediate: $\mathrm{OR} 1.15,95 \% \mathrm{Cl} 1.03$ to $1.28, \mathrm{p}=0.011$; managerial/professional: NS; in prison: NS) and prescription charges (reference: exemption; not exempt: OR 1.15 $95 \% \mathrm{Cl} 1.10$ to $1.21, \mathrm{p}<0.001)$.

$\dagger$ Due to the nature of a telephone intervention, $\mathrm{CO}$ validation was not attempted in most cases so this result would not be reliable and is not presented.

Strata $=1$, cluster $=24$, sampling design $\mathrm{df}=23$.

CO, carbon monoxide; NRT, nicotine replacement therapy; SSS, stop smoking service. 


\section{DISCUSSION}

We found that smokers attending groups run by specialists using combination NRT or varenicline have a better chance of success than those being treated in primary care with one-to-one support and use of single NRT. Thus findings from RCTs ${ }^{6}$ appear to translate well into clinical practice. However, the most effective forms of intervention are uncommon. ${ }^{3}$ It may be argued that this reflects smoker preferences. However, many services do not offer the optimal options. ${ }^{3}$ Smokers using the more successful intervention options tend to be more dependent, so higher success rates are unlikely to be due to an 'easier' clientele.

A potential limitation is that we sampled only a subset of SSSs. However, the characteristics of the smokers and interventions were similar to those of all SSSs. Another limitation is the reliance on 4-week quit data as the outcome measure. However, long-term success rates can be predicted using short-term success. Bias could arise from services with poorer follow-up also being more likely to use intervention options that we found to be less effective. Against this, the 4-week session is part of the intervention ${ }^{6}$ and the findings remained even after adjusting for SSS as a predictor (data not shown). The effects of other factors such as motivational differences cannot be entirely ruled out.

The analyses did not take account of the possibility that some smokers contributed more than one treatment episode to the database. However, analyses restricted to first treatment episodes have very similar results, with pharmacy and primary care settings performing worse than specialist clinics (see table S2 in online supplement).

One possible reason for a failure to optimise the quality of the intervention is a focus on sheer numbers of 4-week quitters rather than numbers of smokers actually helped to quit. If we assume that $25 \%$ of clients would have succeeded for 4 weeks with just medication, ${ }^{6}$ a strong case could be made that services should be judged on the numbers of 4-week quitters generated over and above this (subject to some variations according to the client group).

In summary, a number of service delivery characteristics are associated with higher success rates. There is a significant opportunity for commissioners and providers to increase provision of the optimum service and preserve many more lives.

Acknowledgements lan Baker at North51 collated and transferred the raw data. David Boniface ran additional analyses.
Funding The study was funded by Cancer Research UK (grant number CR-UK C1417/A7972) and the Department of Health (DOH T336/BSS/M award number 49945).

Competing interests RW undertakes research and consultancy for companies that develop and manufacture smoking cessation medications (Pfizer, J\&J, McNeil, GSK, Nabi, Novartis and Sanofi-Aventis). He also has a share of a patent for a novel nicotine delivery device and is a trustee of QUIT, a charity that provides stop smoking support. $\mathrm{EC}$ receives royalties from a book on smoking cessation. AMcE receives a personal income from Cancer Research UK via University College London. He has received travel funding, honoraria and consultancy payments from manufacturers of smoking cessation products (Pfizer, J\&J, McNeil, GSK, Nabi, Novartis and Sanofi-Aventis). He also receives payment for providing training to smoking cessation specialists, receives royalties from books on smoking cessation and has a share in a patent of a nicotine delivery device.

Ethics approval Joint UCL, UCLH and Royal Free Biomedical research Unit.

Contributors LSB cleaned and analysed the data, drafted and revised the paper. RW provided the idea for the study, initiated the collaboration with the data provider North51, secured funding for this study, led the design, contributed to the data analysis and revised the draft paper. He is guarantor for this study. MSMcD contributed to the design and data analysis, drafted and revised the paper. JAF contributed to the design and data analysis and revised the draft paper. EC helped to devise the study, secured the data and commented on a draft of the paper. AMcE secured funding for this study and revised the draft paper. All authors had full access to all of the data (including statistical reports and tables) in the study and can take responsibility for the integrity of the data and the accuracy of the data analysis. All authors approved the final version of the article.

Provenance and peer review Not commissioned; externally peer reviewed.

\section{REFERENCES}

1. West R, Shahab L. Smoking cessation interventions. In: Killoran A, Kelly M, eds. Evidence-Based Public Health Effectiveness and efficiency. Oxford: University Press, 2009:215-32.

2. National Institute for Health and Clinical Excellence (NICE). Smoking Cessation Services in Primary Care, Pharmacies, Local Authorities and Workplaces, Particularly for Manual Working Groups, Pregnant Women and Hard to Reach Communities. London: NICE, 2008.

3. The NHS Information Centre. Statistics on NHS Stop Smoking Services: England, April 2009-March 2010. Leeds: NHS Information Centre, 2010. http://www.ic.nhs. uk/webfiles/publications/Health and Lifestyles/SSS_2009_10_revised.pdf.

4. Judge $\mathbf{K}$, Bauld L, Chesterman J, et al. The English smōking treatment services: short-term outcomes. Addiction 2005;100(Suppl 2):46-58.

5. Borland R, Yong HH, Balmford J, et al. Motivational factors predict quit attempts but not maintenance of smoking cessation: findings from the International Tobacco Control Four country project. Nicotine Tob Res 2010;12(Suppl):S4-11.

6. Department of Health. NHS Stop Smoking Services - Service and Monitoring Guidance. London: Department of Health, 2010. http://www.dh.gov.uk/en/ Publicationsandstatistics/Publications/PublicationsPolicyAndGuidance/DH_109696.

7. Heatherton TF, Kozlowski LT, Frecker RC, et al. The Fagerstrom Test for Nicotine Dependence: a revision of the Fagerstrom Tolerance Questionnaire. Br J Addict 1991;86:1119-27. 